\title{
Borrelia recurrentis Characterization and Comparison with Relapsing-Fever, Lyme-Associated, and Other Borrelia spp.
}

\author{
S. J. CUTLER, ${ }^{1 *}$ J. MOSS,${ }^{2}$ M. FUKUNAGA, ${ }^{3}$ D. J. M. WRIGHT, ${ }^{1}$ \\ D. FEKADE, ${ }^{4}$ AND D. WARRELL ${ }^{5}$ \\ Department of Medical Microbiology ${ }^{1}$ and Department of Histopathology, ${ }^{2}$ Charing Cross Hospital, \\ London, W6 8RF, and Centre for Tropical Medicine, University of Oxford, John Radcliffe \\ Hospital, Oxford, ${ }^{5}$ United Kingdom; Department of Molecular Microbiology, \\ Fukuyama University, Hiroshima, Japan ${ }^{3}$; and Department of Internal \\ Medicine, Black Lion Hospital, Addis Ababa, Ethiopia ${ }^{4}$
}

\begin{abstract}
Borrelia recurrentis, the cause of louse-borne relapsing fever, has until recently been considered noncultivable, which has prevented characterization of this spirochete. We successfully cultivated 18 strains from patients with louse-borne relapsing fever and present the initial characterization of these isolates. Electron microscopy revealed spirochetal cells with pointed ends, an average wavelength of $1.8 \mu \mathrm{m}$, an amplitude of 0.8 $\mu \mathrm{m}$, and 8 to 10 periplasmic flagella. The $G+C$ ratio was $28.4 \mathrm{~mol} \%$. Whole DNA-DNA hybridizations showed similarity between the isolates of $B$. recurrentis but not with Borrelia hermsii, Borrelia parkeri, Borrelia turicatae, or the Lyme-associated borreliae. Sequencing studies of both the flagellin and 16S RNA genes revealed that the greatest similarity was between $B$. recurrentis and Borrelia duttonii. Analysis of the sodium dodecyl sulfatepolyacrylamide gel electrophoresis profiles of strains revealed four groups based on the position of a major protein band (one of the groups showed some heterogeneity and was subdivided into four subgroups). Pulsed-field gel electrophoresis revealed five distinct patterns.
\end{abstract}

In the last century, successive epidemics of louse-borne relapsing fever spread from Asiatic Russia into Europe, causing death in from 2 to $40 \%$ of the cases. Now, this disease has all but vanished in all areas except the Andean foothills, the Ethiopian highlands, and nearby Rwanda. Even in the turmoil in the former Yugoslavia, the appearance of louse-borne typhus was not accompanied by relapsing fever. Why the disease has persisted in only a few isolated foci and has become invisible elsewhere remains an enigma.

The vector of this disease is the infected human body louse (Pediculus humanus humanus). Lice become infected by feeding on a spirochetemic patient. The ingested spirochetes enter the gut and cross the epithelium into the hemolymph. Transmission to humans occurs by contamination of abraded skin

TABLE 1. Strains used in this study

\begin{tabular}{lll}
\hline \multicolumn{1}{c}{ Organism } & \multicolumn{1}{c}{ Strain(s) } & \multicolumn{1}{c}{ Location } \\
\hline B. recurrentis & A1 to A18 & Addis Ababa, Ethiopia \\
B. burgdorferi sensu stricto & B31 & United States \\
B. garinii & 20047 & France \\
B. afzelii & VS461 & France \\
B. japonica & HO14 & Japan \\
Group VS116 & UK & United Kingdom \\
Group PotiB2 & PotiB2 & Portugal \\
B. miyamotoi & HT31 & Japan \\
B. hermsii & HS1 & United States \\
B. parkeri & & United States \\
B. turicatae & & United States \\
\hline
\end{tabular}

${ }^{a}$ Strain A1 has been deposited in the American Type Culture Collection as strain ATCC 700241

* Corresponding author. Mailing address: Department of Medical Microbiology, Charing Cross Hospital, Fulham Palace Road, London, W6 8RF, United Kingdom. Phone: 01818467570 . Fax: 01818467261. E-mail: rabm001@cxwms.ac.uk. with hemolymph from a crushed infected louse. Clinically, louse-borne relapsing fever is characterized a 5- to 7-day incubation period, one to five relapses of fever, and spirochetemia (notably fewer spirochetemias than in tick-borne relapsing fever). Febrile episodes typically last 2 to 9 days, with an afebrile interval of about 9 days. The initial fever is usually the longest and most severe and is followed by milder relapses. Very high spirochete counts are usually associated with a poorer prognosis. Mortality is higher in louse-borne relapsing fever (up to $40 \%$ in untreated cases and $10 \%$ in treated cases) than in the tick-borne variety; this may be related to higher counts of circulating spirochetes. It is possible that as seen in outbreaks, there is a spectrum of severity.

We were able to culture Borrelia recurrentis for the first time since it was described in 1867 by Obermeier (12). Preliminary characterization of $B$. recurrentis and a comparison of this organism with relapsing-fever, Lyme-associated, and other borrelial species are described in this paper.

TABLE 2. B. recurrentis strains grouped according to plasmid patterns and protein profiles

\begin{tabular}{|c|c|c|c|c|c|c|c|}
\hline \multirow[b]{2}{*}{$\begin{array}{l}\text { Plasmid } \\
\text { group }\end{array}$} & \multicolumn{7}{|c|}{ Protein profile(s) } \\
\hline & $\begin{array}{c}\text { Protein } \\
\text { group } \\
1\end{array}$ & $\begin{array}{c}\text { Protein } \\
\text { group } \\
2\end{array}$ & $\begin{array}{c}\text { Protein } \\
\text { group } \\
3\end{array}$ & $\begin{array}{l}\text { Protein } \\
\text { subgroup } \\
4 a\end{array}$ & $\begin{array}{l}\text { Protein } \\
\text { subgroup } \\
4 \mathrm{~b}\end{array}$ & $\begin{array}{l}\text { Protein } \\
\text { subgroup } \\
\quad 4 c\end{array}$ & $\begin{array}{l}\text { Protein } \\
\text { subgroup } \\
4 \mathrm{~d}\end{array}$ \\
\hline Type 1 & $\begin{array}{c}\mathrm{A} 1, \mathrm{~A} 2, \\
\mathrm{~A} 3\end{array}$ & A5 & & & & & \\
\hline Type 2 & A4 & & $\begin{array}{l}\mathrm{A} 6, \mathrm{~A} 7 \\
\mathrm{~A} 8, \mathrm{~A} 9\end{array}$ & $\mathrm{~A} 10$ & & & \\
\hline Type 3 & & & & & $\mathrm{~A} 11, \mathrm{~A} 12$ & & \\
\hline Type 4 & & & & & & $\begin{array}{l}\mathrm{A} 13, \mathrm{~A} 14, \\
\mathrm{~A} 15, \mathrm{~A} 16\end{array}$ & \\
\hline Type 5 & & & & & & & $\mathrm{~A} 17, \mathrm{~A} 18$ \\
\hline
\end{tabular}



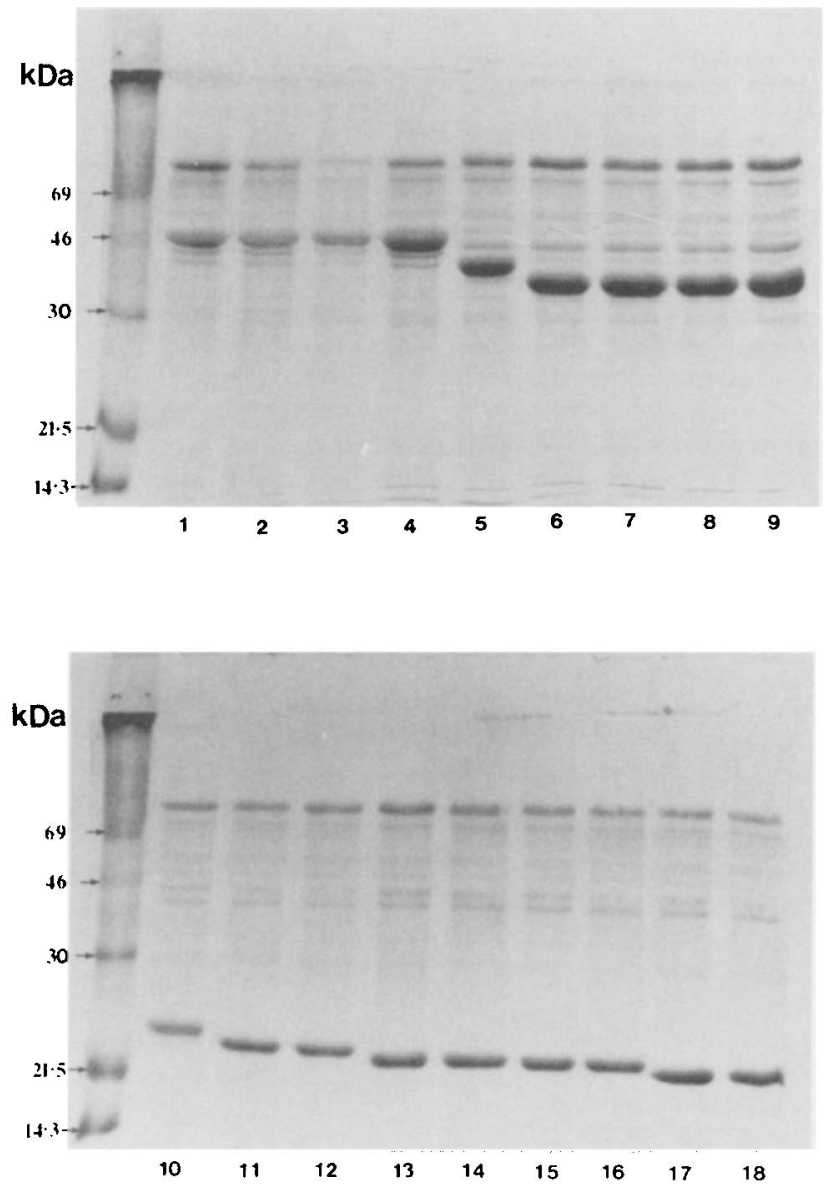

FIG. 1. Coomassie blue-stained SDS-PAGE protein profiles of $B$ recurrentis A1 to A18. Lanes 1 through 4, protein group 1 strains; lane 5, protein group 2 strain; lanes 6 through 9, protein group 3 strains; lane 10 , protein subgroup 4 a strain; lanes 11 and 12, protein subgroup 4b strains; lanes 13 through 16, protein subgroup $4 \mathrm{c}$ strains; lanes 17 and 18 , protein subgroup $4 \mathrm{~d}$ strains.

\section{MATERIALS AND METHODS}

Louse-borne relapsing-fever patients. All patients from which cultures were obtained were demonstrated to be spirochetemic by blood film microscopy by using Wright's stain. One patient was female, and the remaining 17 patients were male. The ages of the patients ranged from $<12$ to 36 years. Most were laborers or jobless. Body lice were found on the clothing in all cases. The duration of clinical symptoms varied between 3 and 7 days.

Bacterial strains and culture conditions. The strains used in this study are listed in Table 1. All isolates were cultured in BSK II medium (3). Relapsingfever strains were maintained by thrice weekly subculturing, and the remaining strains were subcultured on a weekly basis. The strains of Lyme-associated and tick-borne relapsing-fever borreliae were of unknown passage number. The strains of $B$. recurrentis had passage numbers between 8 and 15; this was the minimum number of passages necessary to obtain sufficient material to undertake the study.

Sodium dodecyl sulfate (SDS)-polyacrylamide gel electrophoresis (PAGE). Borrelial cells were harvested from BSK II medium by centrifugation at $3,500 \times$ $g$, washed twice, and suspended in phosphate-buffered saline (Oxoid) to give a protein concentration of approximately $1.8 \mathrm{mg} / \mathrm{ml}$ (as determined with a Bio-Rad Bradford protein assay kit). Cells were diluted in electrophoresis sample buffer containing (final concentrations) $75 \mathrm{mM}$ Tris- $\mathrm{HCl}, 100 \mathrm{mM}$ dithiothreitol, $2 \%$ (vol/vol) SDS, and 10\% (vol/vol) glycerol. This mixture was boiled for $5 \mathrm{~min}$, divided into aliquots, and frozen at $-20^{\circ} \mathrm{C}$ until it was required.

Samples were boiled again for $5 \mathrm{~min}$ immediately before $5-\mu l$ volumes were loaded into wells on minigels (type SE250 Mighty Small system; LKB, Ltd.). The optimal sample concentration for gel loading was determined by dilution. Borrelial proteins were stacked by using a $4 \%$ stacking gel and then were separated by discontinuous SDS-PAGE by using $12.5 \%$ gels run at $150 \mathrm{~V}$ (gels were prepared from a $30 \%$ [wt/vol] acrylamide $-0.8 \%$ [wt/vol] bisacrylamide stock solution [Protogel; Flowgen Instruments, Ltd.]).

\section{$\begin{array}{llllllllllllllll}1 & 2 & 3 & 4 & 5 & 6 & 7 & 8 & 9 & 10 & 11 & 12 & 13 & 14 & 15\end{array}$}

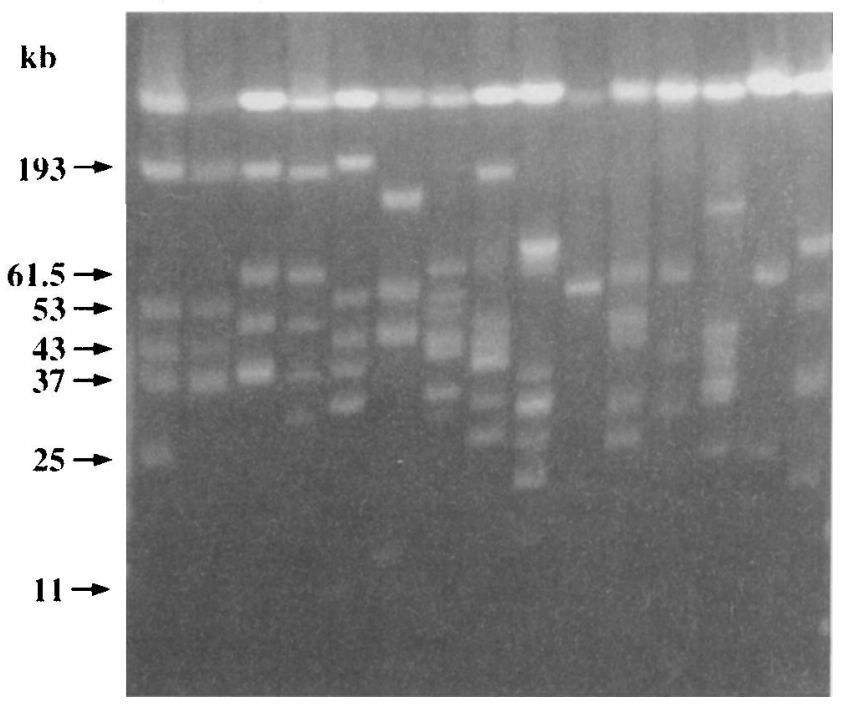

FIG. 2. PFGE patterns comparing each plasmid type of $B$. recurrentis with tick-borne and Lyme-associated borreliae. Lane $1, B$. recurrentis type 1 ; lane $2, B$. recurrentis type 2 ; lane $3, B$. recurrentis type 3 ; lane $4, B$. recurrentis type 4 ; lane $5, B$. recurrentis type 5 ; lane $6, B$. hermsii; lane $7, B$. parkeri; lane $8, B$. turicatae; lane $9, B$. miyamotoi; lane $10, B$. burgdorferi sensu stricto; lane $11, B$. garinii; lane 12 , B. afzelii; lane 13, B. japonica; lane 14, B. burgdorferi UK (group VS116); lane 15, B. burgdorferi PotiB2.

Immunoblotting. SDS-PAGE gels as described above were blotted onto nitrocellulose membranes as described elsewhere (11). After these membranes had been blocked overnight in 5\% dried milk (Marvel) in Tris-buffered saline (50

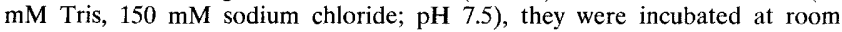
temperature overnight with 1:1,000 dilutions of monoclonal antibody H9724 (4). After washing, each membrane was incubated with a 1:3,000 dilution of goat anti-mouse alkaline phosphatase conjugate (Bio-Rad), washed, and developed by using a solution containing $p$-nitroblue tetrazolium chloride $(0.3 \mathrm{mg} / \mathrm{ml})$ and 5-bromo-4-chloro-3-indolylphosphate $p$-toluidine salt $(0.15 \mathrm{mg} / \mathrm{ml})$, and the reaction was stopped by washing in distilled water (11). The molecular weight of the flagellin band could then be determined by comparison to molecular weight standards (Rainbow markers; Amersham International).

Coomassie blue staining. Minigels were stained by using a Coomassie blue stain (2.5 g of Coomassie blue R250 in 1 liter of $95 \%$ [vol/vol] ethanol, filtered before use)-10\% (vol/vol) glacial acetic acid solution (1:1) overnight. The gels were destained twice for $1.5 \mathrm{~h}$ with $100 \mathrm{ml}$ of $95 \%$ (vol $/ \mathrm{vol}$ ) ethanol and $150 \mathrm{ml}$ of $5 \%$ (vol/vol) glacial acetic acid, then with $33 \mathrm{ml}$ of $95 \%$ (vol/vol) ethanol and $133 \mathrm{ml}$ of $5 \%$ (vol/vol) glacial acetic acid for $2 \mathrm{~h}$, and finally with $117 \mathrm{ml}$ of $5 \%$ (vol/vol) glacial acetic acid and $50 \mathrm{ml}$ of distilled water overnight.

Pulsed-field gel electrophoresis (PFGE). Cells were harvested from BSK II medium by centrifugation at $3,500 \times g$ for $20 \mathrm{~min}$, washed twice with a wash solution ( $75 \mathrm{mM} \mathrm{NaCl}, 25 \mathrm{mM}$ EDTA; pH 7.5), and resuspended in $1 \mathrm{ml}$ of the wash solution. The optical density at $600 \mathrm{~nm}$ was adjusted to 2 . Blocks were prepared by using a 1:1 dilution of cells and $1 \%$ (vol/vol) low-melting-point agarose (Promega) poured into $100-\mu \mathrm{l}$ molds. DNA was ext mcted by incubation at $50^{\circ} \mathrm{C}$ overnight with $1 \mathrm{ml}$ of $0.5 \mathrm{M}$ EDTA (pH 8.5)-1\% (wt/vol) sarcosylproteinase $\mathrm{K}(10 \mu \mathrm{g} / \mathrm{ml})$ per block. Following extraction, the blocks were washed four times with TE (10 mM Tris-Cl [pH 7.6], $1 \mathrm{mM} \mathrm{EDTA})$ and stored at $4^{\circ} \mathrm{C}$ in TE.

Two-hundred-milliliter $1.2 \%$ agarose gels were loaded with blocks, sealed with agarose, and run in $0.5 \%$ TBE $(0.045 \mathrm{M}$ Tris-borate, $0.001 \mathrm{M}$ EDTA) by using a Pharmacia Gene Navigator. Lambda ladder, delta 39 (Promega), 8- to 48-kb,

TABLE 3. Molecular sizes of B. recurrentis plasmids

\begin{tabular}{|c|c|}
\hline $\begin{array}{l}\text { Plasmid } \\
\text { group }\end{array}$ & Plasmid sizes $(\mathrm{kb})$ \\
\hline $\begin{array}{l}\text { Type } 1 . \\
\text { Type } 2 . \\
\text { Type } 3 . \\
\text { Type } 4 . \\
\text { Type } 5 .\end{array}$ & $\begin{array}{l}\ldots 193,53,43,37,25,11 \\
\ldots 192,53,43,37,11 \\
.185,61.5,46,37,11 \\
.183,61.5,46,37,29,11 \\
.194,53,43,37,31,11\end{array}$ \\
\hline
\end{tabular}



ATGATCATAAATCATARTACGTCAGCTATAAATGCTTCAAGAAATAATGCTATTAATGT ATGATCATAAATCATAATACGTCAGCTATAAATGCTTCAAGAAATAATGCTATTAATGTI TATAAATGCTTCAAGAAATAATGCTATTAATGTT TATAAATGCTTCAAGAAATAATGCTATTAATGTT TATAAATGCTTCAAGAAATAATGCTATTAATGTT ATGATTATAAATCATAATACGTCAGCCATAAATGCTTCCAGAAATAATGGCATTAATGCT ATGATCATAAATCATAATACGTCAGCTATAAATGCTTCAAGAAATAATAGCATTAATGCT ATGATCATAAATCATAATACGTCAGCTATAAATGCTTCAAGAAATAATAGCATTAATGCT ATGATCATAAATCATAATACGTCAGCTATAAATGCTTCAAGGAATAATAGCATTAATGCT TATAAATGCTTCAAGAAATAATAGCATTAATGCT TATTAATGCTTCAAGAAATAATGCCATTAATGCT TATTAATGCTTCAAGAAATAATGGTATTAATGCT ATGATTATCAATCATAATACATCAGCTATTAATGCTTCAAGAAATAATGGCATTAACGCT

120 GCTAATCTTAGCAAAACTCAAGAAAAACTTTCTAGTGGGCATAGAATTAATCGTGCATCT GCTAATCTTAGCAAAACTCAAGAAAAACTTTCTAGTGGGCATAGAATTAATCGTGCATCT GCTAATCTTAGCAAAACTCAAGAAAAACTTTCTAGTGGGCATAGAATTAATCGTGCATCT GCTAATCTTAGCAAAACTCAAGAGAAACTTTCTAGTGGGCATAGAATTAATCGCGCATCT GCTAATCTTAGCAAAACTCAAGAGAAACTTTCTAGTGGGCATAGAATTAATCGCGCATCT GCTAATCTTAGCAAAACTCAAGAAAAACTTTCTAGTGGGTATAGAATTAATCGTGCATCT ACTAATCTTAGCAAAACTCAAGAAAAACTTTCTAGTGGGCATAGAATTAATCGTGCATCT GCTAATCTTAGCAAAACTCAAGAAAAACTTTCTAGTGGGTATAGAATTAATCGTGCATCT GCTAATCTTAGCAAAACTCAAGAAAAACTTTCTAGTGGGCATAGAATTAATCGTGCATCT GCTAATCTTAGAAAAACTCAAGAAAAACTTTCTAGTGGGCATAGAATTAATCGTGCATCT GCTAATCTTAGTAAAACCCAAGAGAAGCTTTCTAGTGGTTACAGAATTAATCGAGCTTCT GCTAATCTTAGTAAAACTCAAGAGAAGCTTTCTAGTGGTTACAGAATTAATAGAGCTTCT GCTAATCTTAGTAAAACTCAAGAAAAGCTTTCTAGTGGGTACAGAATTAATCGAGCTTCT

180

GATGATGCTGCTGGTATGGGTGTTGCTGGGAAAATT AACGCTCAAATTAGAGGGTTATCC GATGATGCTGCTGGTATGGGTGTTGCTGGGAAAATTAACGCTCAAATTAGAGGGTTATCC GATGATGCTGCTGGTATGGGTGTTGCTGGGAAAATTAACGCTCAAATTAGAGGGTTATCC GATGATGCTGCTGGTATGGGTGTTGCTGGAAAAATTAATGCTCAAATTAGAGGATTATCC GATGATGCTGCTGGTATGGGTGTTGCTGGGAAAATTAATGCTCAAATTAGAGGATTATCC GATGATGCTGCTGGTATGGGTGTTGCTGGTAAGCTTAATTCACAAATTAGAGGATTGTCT GATGATGCTGCTGGTATGGGCGTTGCTGGAAAAATTAATGCTCAAATTAGAGGGTTGTCT GATGATGCTGCTGGTATGGGTGTTGCTGGAAAGATTAATGCTCAAATTAGAGGATTATCT GATGATGCTGCTGGAATGGGTGTTGCAGGAAAGATTAATGCTCAAATTAGAGGATTATCT GATGATGCTGCTGGAATGGGTGTTGCAGGAAAGATTAATGCTCAAATTAGAGGATTATCT GATGATGCTGCTGGTATGGGGGTTTCTGGCAAGATTAATGCTCAAATAAGAGGCTTATCA GATGATGCTGCTGGTATGGGGGTTTCTGGGAAGATTAATGCTCAAATAAGAGGTTTATCA GATGATGCTGCTGGCATGGGAGTTTCTGGTAAGATTAATGCTCAAATAAGAGGTTTGTCA

240

CAAGCTTCAAGAAATACTTCAAAGGCGATAAATTTTATTCAAACAACAGAAGGAAATTTG CAAGCTTCAAGAAATACTTCAAAGGCGATAAATTTTATTCAAACAACAGAAGGAAATTTG CAAGCTTCAAGAAATACTTCAAAGGCGATAAATATTATTCACACAACAGAAGGAAATTTG CAAGCTTCAAGAAATACTTCAAAGGCGATAAATTTTATTCAAACAACAGAAGGAAATTTG CAAGCTTCAAGAAATACTTCAAAGGCGATAAATTTTCTTCAAACACCAGAAGGAAATTTG CAAGCTTCTAGAAATACCTCAAAGGCTATAAATTTTATTCAAACAACAGAAGGAAATTTG CAGGCTTCTAGAAATACTTCAAAGGCTATAAATTTTATTCAAACAACAGAAGGAAATTTA CAGGCTTCTAGAAATACTTCAAAGGCTATAAATTTTATTCAAACAACAGAAGGAAATTTG CAGGCTTCTAGAAATACTTCAAAGGCTATAAATTTTATTCAAACAACAGAAGGGAATTTA CAGGCTTCTAGAAATACTTCAAAGGCTATAAATTTTATTCAAACAACAGAAGGGAATTTA CAAGCTTCTAGAAACACTTCAAAAGCTATCAATTTTATTCAGACAACAGAAGGAAATTTA CAAGCTTCTAGAAACACTTCAAAAGCTATTAATTTTATTCAGACAACAGAAGGAAATTTG CAAGCTTCTAGAAATACTTCAAAGGCTATTAATTTTATTCAGACAACAGAAGGGAATTTA
B. rec.

B.dut.

B.cro.

B.his.

B.Spa.

B.miy .

B.her.

B. cor.

B.tur.

B.par.

B.afz.

B. gar.

B.bur.
B. rec.

B.dut.

B.cro.

B.his .

B.spa.

B.miy .

B.her.

B.cor.

B.tur.

B.par.

B.afz.

B.gar.

B.bur.

B. rec.

B.dut,

B.cro.

B.his .

B. Spa .

B.miy.

B.her.

B.cor.

B.tur.

B. par.

B.afz.

B. gar.

B. bur.

FIG. 3. Multiple alignment of flagellin sequences for relapsing-fever, Lyme-associated, and other borreliae. Abbreviations: B.rec., B. recurrentis; B.dut., B. duttonii; B.croc., B. crocidurae; B.his., B. hispanica; B.Spa., Borrelia sp. strain Spain; B.miy., B. miyamotoi; B.her., B. hermsii; B.cor., B. coriaceae; B.tur., B. turicatae; B.par., B. parkeri; B.afz., B. afzelï; B.gar., B. garinii; B.bur., B. burgdorferi; B.lon., B. lonestari.

and 5 -kb ladder pulsed-field markers (Bio-Rad) were included on every gel. The gels were run by using $200 \mathrm{~V}$ with pulse times ranging from 0.5 to $15 \mathrm{~s}$ for a total of $17 \mathrm{~h}$. Bands were visualized following staining with ethidium bromide (1 $\mu \mathrm{g} / \mathrm{ml})$ for $1 \mathrm{~h}$

DNA sequencing. The 16S rRNA gene was sequenced from a range of borrelial strains, including $B$. recurrentis $\mathrm{A} 1$. The sequences obtained were compared by using a range of phylogenetic techniques, and the results have been published elsewhere (26). Similarly, the flagellin gene was amplified, ligated into an Escherichia coli vector, and sequenced by using the methods of Fukunaga et al. (15, 16). Multiple alignments were prepared by using the Clustal program, and a phylogenetic analysis was performed by using the neighbor-joining method in the DNA STAR software package. 
GATGAAGTAGAGAAAGTGTTGGTGAGAATGAAAGAGCTTGCTGTTCAATCTGGTAATGGT GATGAAGTAGAGAAAGTGTTGGTGAGAATGAAAGAGCTTGCTGTTCAATCTGGTAATGGT GATGAAGTAGAGAAAGTGTTGGTGAGAATGAAAGAGCTTGCTGTTCAATCTGGTAATGGT GATGAAGTAGAAAAAGTGTCGGTGAGAATGAAGGAGCTCGCGGTTCAATCTGGTGGGGGT GCTGAAGTAGAGANAGTGTTGGTGAGAATGAAAGAGGTTGCTGCTCAATCTGGTAATGGT AACGAGGTAGAGAAAGTATTAGTAAGAATGAAAGAACTTGCTGTTCAGTCTGGTAATGGT AATGAAGTAGAGAGAGTATTAGTAAGAATGAAAGAACTTGCTGTTCAATCTGGTAATGGT GATGAAGTAGAGAAAGTATTAGTAAGAATGAAAGAACTTGCTGTTCAGTCTGGTAATGGT AATGAAGTAGAGAAAGTATTAGTAAGAATGAAAGAACTTGCTGTGCAGTCTGGTAATGGT AATGAAGTAGAGAAAGTATTAGTAAGAATGAAAGAACTTGCTGTTCAGTCTGGTAATGGT AATGAAGTAGAAAAAGTTTTAGTAAGAATGAAAGAATTAGCAGTTCAATCAGGTAACGGA AATGAAGTAGAAAAAGTTTTAGTAAGAATGAAAGAATTAGCAGTTCAATCAGGTAACGGT AATGAAGTAGAAAAAGTCTTAGTAAGAATGAAGGAATTGGCAGTTCAATCAGGTAACGGC

ACATATTCAGATGCAGACAGAGGTTCTATTCAGATTGAAGTTGAACAACTTACAGATGAG ACATATTCAGATGCAGACAGAGGTTCTATTCAGATTGAAGTTGAACAACTTACAGATGAG ACATATTCAGATGCAGTCAGAGGTTCTATTCAGATTGAAGTTGAACAACTTACAGATGAG ACATATTCAGATGCAGACAGGGGTTCTACTCAGAGTGAAGATGAACAACTCACAGATGAG ACATATTCAGATGCAGACAGAGGTTCTATTCAGATTGAAATTGAGCAACTTACAGATGAG ACATACTCAGATTCAGATAGAGGGTCTATTCAGATTGAAATTGAACAACTTACAGATGAA ACATATTCAGATGCAGACAGAGGTTCTATTCAAATTGAAATTGAACAACTTACAGATGAA ACATATTCAGATGCAGACAGAGGTTCTATTCAAATTGAAATTGAGCAACTTACAGATGAA ACATATTCAGATGCAGACAGAGGTTCTATTCAAATTGAGATTGAGCAACTTACAGATGAA ACATATTCAGATGCAGACAGAGGTTCTATTCAAATTGAAATTGAGCAACTTACAGATGAA ACATATTCAGATGCAGACAGAGGTTCTATTCAAATTGAAATTGAGCAACTTACAGATGAA ACGTATTCAGACTCAGACAGAGGTTCTATACGAATTGAAATAGAGCAACTTACAGACGAA ACATATTCAGACGCAGACAGAGGTTCTATACAAATTGAAATAGAGCAACTTACAGACGAA ACATATTCAGATGCAGACAGAGGTTCTATACAAATTGAAATAGAGCAACTTACAGACGAA

B.rec.

B. dut.

B.cro.

B.his.

B.Spa.

B.miy.

B.lon.

B.her.

B.cor.

B.tur.

B.par.

B.afz.

B.gar.

B.bur.

420

ATCAATAGAATTGCTGATCAAGCACAATATAACCATATGCATATGTTATCTAATAGATCA ATCAATAGAATTGCTGATCAAGCACAATATAACCATATGCATATGTTATCTAATAGATCA ATCAATAGAATTGCTGATCAAGCACAATATAACCATATGCATATGTTATCTAATAGATCA CTCAATAGAATTGCTGATCAAGCACNATATAACCATATCCATNTGTTATCAAATAGATCA ATCAATAGAATTGCTGATCAGGCACAATATAACCATATGCATATGTTATCTAATAGATCA ATAAACAGAATTGCTGATCAGGCTCAATACAACCAAATGCATATGTTATCTAATAAGTCA ATTAACAGAGTTGCTGATCAGGCTCAATACAACCAGATGCATATGTTATCTAACAAATCA ATCAACAGAATTGCTGATCAGGCTCAATACAACCAAATGCATATGTTGTCCAACAAGTCA ATTAACAGAATTTCTGATCAAGCTCAATACAATCAAATGCATATGTTGTCTAATAAATCA ATCAACAGAATTGCTGATCAAGCTCAATACAACCAAATGCATATGTTGTCCAACAAGTCA ATCAACAGAATTGCTGATCAAGCTCAATACAACCAAATGCATATGTTGTCCAATAAGTCA ATTAATAGAATTGCTGATCAGGCTCAATATAACCAAATGCACATGTTGTCAAACAAATCT ATTAATAGAATTGCTGATCAAGCTCAATATAACCAAATGCACATGTTGTCAAACAAATCT ATTAATAGAATTGCTGATCAAGCTCAATATAACCAAATGCACATGTTATCAAACAAATCT

B.rec. B. dut.

B.cro.

B.his .

B.Spa.

B.miy.

B.lon.

B.her.

B.cor.

B.tur.

B.par.

B. afz.

B.gar.

B.bur.

480

TCTGCTGAGCATGTAAGAACAGCTGAAGAGCTTGGAATGCAACCTGTAAAGATTAATACA TCTGCTGAGCATGTAAGAACAGCTGAAGAGCTTGGAATGCAACCTGTAAAGATTAATACA TCTGCTGAGCATGTAAGAACAGCTGAAGAGCGTGGAATGCAACCTGTAAAGATTAATACT CCTGCTGAGAATGTAAGAACAGCTGGAGAGCTTGGAATGCAACCTGGGAAGATTAATACA GCTGCTGAGAATGTAAGAACAGCTGAAGAGCTTGGAATGCAACCTGTAAAGATTAATACA GCTGCTCAAAATGTAAAAACTGCTGAAGAGCTTGGAATGCAACCTGCAAAAATTAACACA TCTGCTCAAAATGTAAAAACTGCTGAAGAGCTTGGAATGCAACCTGCAAAAATTAATACA GCTGCTCAAAATGTAAAAACAGCTGAAGAGCTTGGAATGCAACCTGCAAAAATTAACACA GCTGCTGAGAATGTAAAAACAGCTGAAGAGCTTGGAATGCAACCTGCAAAAATTAACACA GCTGCTCAAAATATAAAAACAGCTGAAGAGCTTGGAATGCAACCTGCAAAAATTAACACA GCTGCTCAGAATATAAAAACAGCTGAAGAGCTTGGAATGCAACCTGCAAAAATTAACACA GCTTCCCAAAATGTAAAAACAGCTGAAGAGCTTGGAATGCAGCCTGCAAAAATTAACACA GCTTCCCAAAATGTAAGAACAGCTGAAGAACTTGGAATGCAACCTGCAAAAATCAACACA GCTTCTCAAAATGTAAGAACAGCTGAAGAGCTTGGAATGCAGCCTGCAAAAATTAACACA 
540

CCAGCATCATTATCTGAATCACAAGCTTCATGGACATTAAGAGTACATGTTGGTGCAAAT CCAGCATCATTATCTGGATCACAAGCTTCATGGACATTAAGAGTACATGTTGGTGCAAAT CCAGCNTCATTATCTGGATCAGAAGATTCGTGGGGAGTAAGAGTACATGTTGGTGCAAAT CCAGGATCACTATCTGGGTCACAAGCTTCATGGACATTAAGAGTACATGTTGGTGCAAAT CCAGCATCATTATCTGGCTCACAGGCTTCATGGACATTAAGAGTACATGTTGGTGCAAAT CCAGCATCATTGGCTGGATCACAAGCTTCATGGACATTGAGAGTGCATGTAGGTGCAAAT CCAGCATCACTAACTGGAGCACAAGCTTCATGGACATTGAGAGTTCAGGTAGGTGCAAAT CCAGCATCACTAGCTGGATCACAAGCTTCATGGACATTGAGAGTACATGTGGGCGCAAAT CCAGCATCATTAGCTGGCTCACAAGCTTCATGGACATTGAGAGTACAAGTGGGAGCAAAT CCAGCATCATTAGCTGGATCACAAGCTTCATGGACATTAAGAGTACATGTGGGTGCAAAT CCAGCATCATTAGCTGGAGCACAAGCTTCATGGACATTAAGAGTACATGTGGGTGCAAAT CCAGCATCACTTTCAGGATCTCAAGCTTCTTGGACTTTAAGAGTTCATGTGGGAGCAACT CCAGCGTCACTTTCAGGATCTCAAGCTTCTTGGACCTTAAGAGTTCATGTGGGAGCAAAT CCAGCATCACTITCAGGGTCTCAAGCGTCTTGGACTTTAAGAGTTCATGTTGGAGCAAAC

600

CAGGATGAAGCAATTGCTGTTAATATTTATGCAGCTAATGTTGCAAATCTTTTTTCAGGT CAGGATGAAGCAATTGCTGTTAATATTTATGCAGCTAATGTTGCAAATCTTTTTTCAGGT CAGGATGAAGCAATTGCTGTTAATATTTATGCAGCTAATGTTGCAAATCTTTTTTCAGGT CAAGATGAAGCAATTGCTGTTAATATTTATGCAGCTAATGTTGCAGATCTTTTTTCAGGT CAAGATGAAGCAATTGCTGTTAATAATTATGCAGCTAATGTTGCAAATCTTTTTTCAGGT CAGGATGAAGCAATTGCTGTCAATATTTATGCAGCTAATGTTGCAAATCTTTTTAATGGA CAGGATGAAGCAATTGCTGTTAATATTTTCTCAACTAATGTTGCAAATCTTTTTGGTGGA CAGGATGAGGCAATTGCTGTTAATATTTATGCATCTAATGTTGCAAATCTTTTTGCAGGT CAGGACGAAGCAATTGCTGTTAATATTTTTGCATCTAATATTGCAAATCTTTTTGCAGGT CAGGATGAAGCAATTGCTGTTAATATTTATGCAGCTAATGTTGCAAACCTTTTTGCAGGT CAGGATGAAGCAATTGCTGTTAATATTTATGCATCTAATGTTGCAAACCTTTTTGCAGGT CAAGATGAAGCAATTGCTGTAAATATTTATGCAGCTAATGTTGCAAATCTTTTTGCTGGT CAAGATGAAGCGATTGCTGTAAATATTTATGCTGCTAATGTTGCAAATCTATTCTCTGGT CAAGATGAAGCTATTGCTGTAAATATTTATGCAGCTAATGTTGCAAATCTTTTCTCTGGT

660 GAGGGTGCTCAA-----CAAGTAGCTCCAGCTCAAGAGGGTGCACAGCAAGAAGGAGCA GAGGGTGCTCAA-----CAAGTAGCTCCAGCTCAAGAGGGTGCACAGCAAGAAGGAGCA GAGGGTGCTCAA-----CAAGTAGCTCCAGCTCAAGAGGGTGCACAGCAAGAAGGAGCA GAGGGTGCTCAA-----CAAGTAGCTCCAGCTCAAGAGGGTGCACAACAAGAAGGAGGA GAGGGTGCTCAA-----CAAGTAGCTCCAGCTCAAGAGGGTGCACAACAAGAAGGAGCA GAAGGTGCTCAA------_GAGCTCCAGCTCAAGAGGGAGCACAACAGGAGGGAGTT GAAGGTGTTCAA--_-_-_GCGGCTCCAGCTCAAGAGGGTGCACAACAGGAGGGAGTT GAAGGCGCTCAG---_--GCTGCTCCAGTGCAAGAGATAGGACAGCAAGAGGAAGGT GAAGGTGCTCAG-_-_-_-_CTGCTGTAGCT-_-GAAGGTGCACAAGAAGAAGGAGTT GAAGGTGCGCAG-_-_-_-OTTTCTCCAGCTCAGGAAGGTGCACAACAAGAGGGAGTT GAAGGTGCGCAG-_-_-_-_GTTTCTCCAGCTCAGGAAGGTGCACAACAAGAGGGAGTT GAGGGAGCTCAAGCTGCTCAGGCTGCACCTGTTCAAGAGGGTGCTCAAGAAGAAGGAGCT GAAGGAGCTCAGGCTGCTCAGACTGCACCTGTTCAAGAAGGAGCTCAACAAGAAGGAGCT GAGGGAGCTCAAACTGCTCAGGCTGCACCGGTTCAAGAGGGTGTTCAACAGGAAGGAGCT

720

CAAGCAGCTCCAGCTCCAGCAGCAGCTCCAGCTCAAGGTGGAGTTAACTCTCCAGTTAAT CAAGCAGCTCCAGCTCCAGCAGCAGCTCCAGCTCAAGGTGGAGTTAACTCTCCAGTTAAT CAAGCAGCTCCAGCTCCAGCATCAGCTCCAGCTCAAGGTGGAGTTAACTCTCCAGTTAAT CAAGCAGCTCCGCCTCCAGCAGCAGCTCCAACTCAAGGTGGGGTTAACTCTCCAGTTAAT CAAGCAACTCC---TCCAGCAGCAGCTCCAACTCAAGGTGGAGTTAACTCTCCAGTTAAT CAAGCAGTTCCAGCTCCAGCAGCCGCTCCAGTGCAAGGTGGAGTTAATTCTCCAATTAAT CAACC--_-_-_-_-- AGCTCCAGCTCAAGGTGGGATTAGCTCTCCAATTAAT CAAGCAGCTCCAGCTCCAGCAGCAGCTCCAGCTCAAGGTGGAGTTAATTCCCCAATTAAT CAAGCTGGACAAGGTGCAGTAGCAGCTCCAGCTCAAGGTGGAGTTAATTCTCCAATTAAT CAAGCTGCTCCAGCACCAGCAGCAGCTCCAGCTCAAGGTGGAGTTAATTCTCCAGTTAAT CAAGCTGCTCCAGCACCAGCAGCAGCTCCAGCTCAAGGTGGAGTTAATTCACCAGTTAAT CAG-CAA--CCAACACCTGCTACAGCACCTACTCAAGGTGGAGTTAATTCTCCTGTTAAT CAA-CAA--CCAGCACCTGTTACAGCGCCTTCTCAGGGTGGAGTTAATTCTCCTGTTAAT CAA-CAG--CCAGCACCTGCTACAGCACCTTCTCAAGGCGGAGTTAATTCTCCTGTTAAT
B.rec.

B.dut.

B.cro.

B.his.

B.Spa.

B.miy.

B.lon.

B. her.

B.cor.

B.tur.

B.par.

B. afz.

B.gar.

B.bur.

B.rec.

B.dut.

B. cro.

B.his .

B.Spa.

B.miy.

B.lon.

B.her.

B.cor.

B.tur.

B. par.

B. afz.

B. gar.

B. bur.

B. rec.

B.dut.

B.cro.

B.his.

B.Spa.

B.miy.

B.Ion.

$B$.her.

B. cor.

B.tur.

B. par.

B.afz.

B.gar.

B.bur.

B.rec

B.dut.

B.cro.

B.his.

B.Spa.

B.miy.

B. Ion.

B.her.

B.cor.

B.tur.

B.par.

B.afz.

B. gar.

B. bur. 
GTTACAACTGCTGTTGATGCTAATATGTCACTTACAAAAATAGAAGATGCTATTAGAATC GTTACAACTGCTGTTGATGCTAATATGTCACTTACAAAAATAGAAGATGCTATTAGAATG GTTACAACTGCTGTTGATGCTAATATGTCACTTACAAAAATAGAAGATGCTATTAGAATG GTCACAACTGCTGTTGATGCTAATGTGTCACTTACAAAAATAGAAGATGCTATTAGAATG GTTACAACTGCTGTTGATGCTAATATGTCACTTACAAAAATAGAAGATGCTATTAGAATG GTTACAACTGCTATTGATGCTAATATGTCACTTTCAAAGATCGAAGATGCTATTAGAATG GTTACAACTGCTATTGATGCTAATGCATCGCTTACAAAGATTGAAGATGCTATTAGAATG GTTACAACCGCTGTTGATGCTAATATGTCACTTGCAAAGATAGAAGGTGCTATTAGAATG GTTACAACTGCTGTTGATGCTAATATATCACTTGCAAAGATAGAGGATGCTATCAGAATG GTTACAACTACTATTGATGCTAATATGTCACTTTCAAAGATAGAAAATGCTATTAGAATG GTTACAACTACTGTTGATGCTAATATGTCACTTTCAAAGATAGAAAATGCTATTAGAATG GTTACAACCACAGTTGATGCTAATACATCACTTGCTAAAATAGAAAATGCTATTAGAATG GTTACAACCACAGTTGATGCTAATACATCTCTTGCTAAAATAGAAAATGCTATTAGAATG GTTACAACTACAGTTGATGCTAATACATCACTTGCTAAAATTGAAAATGCTATTAGAATG
B.rec.
B. dut.
B.cro.
B. his.
B.Spa.
B.miy,
B.Ion.
B.her.
B.cor.
B.tur.
B.par.
B. afz.
B.gar.
B.bur.

840

ATAACTGATCAAAGAGCAAATCTTGGTGCTTTCCAAAACAGACTTGAATCTGTTAAAGCT ATAACTGATCAAAGAGCAAATCTTGGTGCTTTCCAAAACAGACTTGAATCTGTTAAAGCT ATAGCTGATCAAAGAGCAAATCTTGGTGCTTTCCAAAACAGACTTGAATCTGTTAAAGCT GTAGCTGATCAAAGAGCAAATCTTGGTGC TTTCCAAAACAGACGTGAATCTATTAAGGCT GTAACTGATCAAAGAGCAAATCTTGGTGCTTTCCCAAACAGACTTGAGTCTATTAAGGCT GTAACTGATCAAAGAGCAAATCTTGGTGCTTTTCAAAATAGACTTGAGTCTGTTAAGGCT GTAACTGATCAAAGAGCAAATCTTGGTGCTTTCCAAAATAGACTTGAGTCTGTTAAAGCT GTAACTGATCAAAGAGCAAATCTTGGTGCTTTCCAAAACAGACTTGAGTCTATTAAGGAT GTAACTGATCAAAGAGCAAATCTCGGTGCTTTCCAAAACAGGCTTGAGTCTATTAAGGAT GTAAGTGATCAAAGAGCAAATCTTGGTGCTTTCCAAAATAGACTTGAGTCTATTAAGGCT GTAAGTGATCAAAGAGCAAATCTTGGTGCTTTCCAAAACAGACTTGAGTCTATTAAAGCT ATAAGTGATCAAAGAGCAAATTTAGGTGCTTTCCAAAATAGACTTGAATCTATAAAGAAT ATAAGTGATCAAAGAGCAAATTTAGGTGCTTTCCAAAATAGACTTGAGTCTATAAAGGAT ATAAGTGATCAAAGGGCAAATTTAGGTGCTTTCCAAAATAGACTTGAATCTATAAAGAAT

B.rec.

B.dut.

B.cro.

B.his.

B.Spa.

B.miy.

B.Ion.

B.her.

B.cor.

B.tur.

B.par.

B.afz.

B. gar.

B. bur.

900

AGCACAGAGTATGCTATTGAAAATTTAAAAGCGTCTTATGCTCAAATTAAAGATGCAACA AGCACAGAGTATGCTATTGAAAATTTAAAAGCGTCTTATGCTCAAATTAAAGATGCAACA AGCACAGAGTATGCTATTGAAAATTTAAAAGCGTCTTATGCTCAAATTAAAGATGCAACA AGCACAGAGTATGCTATTGAAAATTTAGAAGCGTCTTATGCTCAAATTAGAGATGCAGCA AGCACAGAGTATGCTATTGAAAATTTAAAAGCGTCTTATGCTCAAATTAAAGATGCATCA AGCACAGACTATGCTATTGAAAACTTGAAAGCATCTTATGCTCAAGTTAAAGATGCAATA AGCACAGATTATGCTATTGAAAACTTAAAAGCGTCTTATGCTCAAATTAAAGATGCAATA AGTACAGAATATGCTATTGAAAACTTGAAAGCATCATATGCTCAAATTAAAGATGCAACA AGCACAGAATATGCTATTGAAAATTTAAAAGCATCTTATGCTCAAATTAAAGATGCAACA AGCACAGAATATGCTATTGAAAACTTAAAATCATCTTATGCTCAAATTCAAGATGCAACA AGCACAGAATATGCTATTGAAAACTTAAAATCATCTTATGCTCAAATTAAAGATGCAACA AGCACTGAGTATGCTATTGAAAATCTAAAAGCATCTTATGCTCAAATAAAAGATGCTACA AGTACTGAGTATGCTATTGAAAACCTAAAAGCATCTTATGCTCAAATAAAAGATGCTACA AGTACTGAGTATGCAATTGAAAATCTAAAAGCATCTTATGCTCAAATAAAAGATGCTACA

B.rec.

B.dut.

B.cro.

B.his .

B. Spa.

B.miy .

B.lon.

B. her.

B. cor.

B. tur.

B.par.

B. afz .

B. gar.

B. bur.

960

ATGACAGATGAAATTGTAGCATCTACAACAAACAGTATTTTGACACAGTCTGCAATGGCT ATGACAGATGAAATTGTAGCATCTACAACAAACAGTATTTTGACACAGTCTGCAATGGCT ATGACAGATGAAATTGTAGCATCTACAACAAACAGTATTTTGACACAGTCTGCAATGGCT ATGACAGATGATATTGTAGCATCTACAACAAATAGTATTTTGACACAGTCTGCAATGGCT ATGACAGATGAAATTGTAGCATCTACAACAAATAGTATTTTGACACAGTCTGCAATGGCT ATGACAGATGAAATTGTGGCATCTACAACTAACAGCATTTTGACACAATCCGCAATGGCT ATGACAGATGAAATTGTAGCATCTACAACCAACAGTATTTTGACACAATCTGCAATGGCT ATGACAGATGAAGTTGTAGCATCAACAACTCACAGTATTTTGACACAATCTGCAATGGCT ATGACAGATGAAGTTGTGGCATCTACAACTAGCAGCATTTTGACACAATCTGCGATGGCT ATGACAGATGAAATTGTGGCATCTACAACTAACAGCATTTTGACACAATCCGCAATGGCT ATGACAGATGAAATTGTGGCATCTACAACTAACAGCATTTTGACACAATCCGCAATGGCT ATGACAGATGAGGTTGTAGCAGCTACAACTAATAGTATTTTAACTCAATCTGCAATGGCA ATGACAGATGAGGTTGTAGCAGCTACAACTAATAGTATTTTGACACAATCTGCAATGGCA ATGACAGATGAGGTTGTAGCAGCAACAACTAATAGTATTTTAACACAATCTGCAATGGCA

B.rec.

B.dut.

B.cro.

B.his.

B.Spa.

B.miy.

B.Ion.

B. her.

B.cor.

B.tur.

B. par.

B. afz.

B.gar.

B.bur. 


ATGATTGCACAAGCAAATCAAGTACCTCAA
ATGATTGCACAAGCAAATCAAGTACCTCAA
ATGATT
ATGATT
ATGATT
ATGATTGCACAAGCAAATCAAGTGCCTCAATATGTATTGTCATTGCTTAGATAG
ATGATT
ATGATTGCACAAGCAAATCAAGTACCTCAA
ATGATTGCACAAGCAAATCAAGTACCTCAA
ATGATTGCACAAGCAAATCAAGTACCTCAA
ATGATT
ATGATT
ATGATT
ATGATTGCGCAGGCTAATCAAGTTCCCCAATATGTTTTTCATTGCTTAGATAA

FIG. 3-Continued.
B.rec.
B.dut.
B.cro.
B.his.
B.Spa.
B.miy.
B.lon.
B.her.
B.cor.
B.tur.
B.par.
B.afz.
B.gar.
B.bur .

G $+\mathrm{C}$ content of DNA. The $\mathrm{G}+\mathrm{C}$ content of $B$. recurrentis was determined by high-performance liquid chromatography. DNA dissolved in water was denatured by boiling for $5 \mathrm{~min}$ and chilled on ice, and $10 \mu \mathrm{l}(3$ to $5 \mu \mathrm{g})$ of DNA was digested with P1 nuclease (DNA-GC kit; Seikagaku Kogyo, Tokyo, Japan) according to the manufacturer's instructions; $10-\mu$ l volumes of hydrolysate and a standard were analyzed by high-performance liquid chromatography (Shimadzu model LC-10AS instrument fitted with a type AQ-312 column; YMC Co., Ltd. Tokyo, Japan). The mobile phase was $10 \mathrm{mM}$ phosphate buffer ( $\mathrm{pH} 3.5$ ) at a flow rate of $1.5 \mathrm{ml} / \mathrm{min}$.

Comparison of $B$. recurrentis DNA with DNAs from other borreliae. DNA was extracted from cells harvested from BSK II medium by incubating the cells for $3 \mathrm{~h}$ at $56^{\circ} \mathrm{C}$ in lysis buffer containing $10 \mathrm{mM}$ Tris (pH 8), 60 mM EDTA (pH 8), $5 \%$ SDS, and $50 \mu \mathrm{g}$ of proteinase $\mathrm{K}$ per $\mathrm{ml}$. The DNA was extracted with phenol, precipitated with ethanol, and resuspended in water. Following $30 \mathrm{~min}$ of incubation at $37^{\circ} \mathrm{C}$ with RNase A $(2 \mu \mathrm{g} / \mathrm{ml})$, the DNA was again precipitated with ethanol, resuspended in water, and stored at $-20^{\circ} \mathrm{C}$ until it was used.

DNA was quantified and diluted to produce dilution curves ranging from 200 ng to $50 \mathrm{pg}$ for each of the louse-borne relapsing-fever strains and other species of tick-borne and Lyme-associated borreliae. A nylon membrane (Hybond-N Amersham International) was divided into a grid, and $1-\mu$ l volumes of each dilution of DNA were dotted onto the membrane, allowed to dry, and fixed with a UV cross-linker.

B. recurrentis A1 was prepared as a probe by using random prime labelling with fluorescein-11-dUTP (Gene Images kit; Amersham International) according to the manufacturer's instructions. Fluorescein-labelled probe was not stored before use. Biotin-labelled probe from the same strain was also prepared (Photoprobe; Vector Laboratories). Briefly, equal volumes of a solution containing $1 \mu \mathrm{g}$ of denatured DNA per $\mathrm{ml}$ and Photoprobe were mixed, and the mixture was irradiated with a sunlamp for $15 \mathrm{~min}$. Unreacted probe was deprotonated by adding 1 volume of $0.1 \mathrm{M}$ Tris- $\mathrm{HCl}(\mathrm{pH} 9.5)$. The mixture was extracted with butanol twice (discarding the upper organic phase) and precipitated with ethanol, and the pellet was dissolved in sterile distilled water. Biotin-labelled probe was stored at $-20^{\circ} \mathrm{C}$ until it was used.

Nylon membranes were prehybridized for $30 \mathrm{~min}$ to $1 \mathrm{~h}$ at $60^{\circ} \mathrm{C}$ by using 0.125 to $0.3 \mathrm{ml}$ of $5 \times \mathrm{SSC}$ (diluted from a $20 \times \mathrm{SSC}$ stock solution [ $1 \times \mathrm{SSC}$ is $0.015 \mathrm{M}$ sodium citrate plus $0.15 \mathrm{M}$ sodium chloride])-0.1\% (vol/vol) SDS-5\% (wt/vol) dextran sulfate-1/20 dilution of liquid block (supplied with the Gene Images kit) per $\mathrm{cm}^{2}$. Denatured probe was added to the prehybridization buffer $(10 \mathrm{ng} / \mathrm{ml})$, and hybridization was allowed to proceed overnight at $60^{\circ} \mathrm{C}$.

Spent hybridization solution was discarded and replaced with preheated $1 \times$ SSC $-0.1 \%$ SDS ( 2 to $5 \mathrm{ml} / \mathrm{cm}^{2}$ of membrane) for $15 \mathrm{~min}$ at $60^{\circ} \mathrm{C}$. This was replaced with $0.5 \times \mathrm{SSC}-0.1 \%$ (vol/vol) SDS, and the preparation was incubated as described above. Bound probe was detected by using a Gene Images CDP-star detection module (Amersham International) for fluorescein-labelled probe according to the manufacturer's instructions. The preparations were exposed to hyperfilm MP (Amersham International) for $30 \mathrm{~min}$ before being developed Membranes hybridized with photobiotinylated probe were first blocked for $2 \mathrm{~h}$ at $37^{\circ} \mathrm{C}$ with $10 \%$ (wt/vol) dried milk (Marvel) in TBS (0.1 M Tris [pH 7.5], $0.15 \mathrm{M}$ sodium chloride) and then were detected by using Vectastain (Vector Laboratories), washed three times in TBS (10 min each time), and exposed to $p$ nitroblue tetrazolium chloride and 5-bromo-4-chloro-3-indoylphosphate $p$-toluidine salt $(30$ and $15 \mathrm{mg} / \mathrm{ml}$, respectively, in $100 \mathrm{ml}$ of Tris-magnesium chloride buffer [0.1 M Tris, $0.5 \mathrm{mM}$ magnesium chloride; $\mathrm{pH}$ 9.5]). The reaction was stopped by immersing the membrane in distilled water.

Electron microscopy. B. recurrentis A1 was grown in BSK II medium, harvested by centrifugation at $3,500 \times g$ for $20 \mathrm{~min}$, and washed once in phosphate-buffered saline $(3,500 \times \mathrm{g}, 20 \mathrm{~min})$, and the pellet was resuspended and fixed in $3 \%$ (vol/vol) glutaraldehyde in $0.1 \mathrm{M}$ cacodylate buffer, recentrifuged, and incubated at $4^{\circ} \mathrm{C}$ for $1 \mathrm{~h}$. Cells were postfixed in $2 \%$ (wt/vol) osmium tetroxide, dehydrated in ascending grades of ethanol, and embedded in Spurr resin. Ultrathin sections were stained with aqueous uranyl acetate and Reynold's lead citrate prior to examination with an electron microscope.

To prepare for negative staining, the pellet was resuspended (1:9) in either $2 \%$ (vol/vol) aqueous phosphotungstic acid (neutralized with $1 \mathrm{M}$ potassium hydroxide) supplemented with $0.01 \%$ ( $\mathrm{vol} / \mathrm{vol}$ ) glycerol or $1 \%$ ammonium molybdate neutralized with concentrated ammonium hydroxide. One drop of suspension was allowed to air dry at room temperature on carbon-coated Formvar films supported on copper grids prior to examination.

\section{RESULTS}

SDS-PAGE. Coomassie blue-stained gels revealed much heterogeneity in the area at and below $45 \mathrm{kDa}$. The protein patterns divided $B$. recurrentis strains into four groups (Fig. 1). One group (four strains) had a major band at $45 \mathrm{kDa}$; the second group consisted of a single strain with a major band at $40 \mathrm{kDa}$; the third group (four strains) had a predominant band at $35.5 \mathrm{kDa}$; and the remaining nine isolates produced major bands between 21.5 and $24 \mathrm{kDa}$. The last group was subdivided into four subgroups to accommodate strains with identical molecular weights. Subgroup $4 \mathrm{a}$ had one member, subgroup $4 \mathrm{~b}$ was comprised of two strains, subgroup $4 \mathrm{c}$ had four members, and subgroup $4 \mathrm{~d}$ contained two strains (Fig. 1).

Immunoblotting with monoclonal antibody H9724. A band located at $41 \mathrm{kDa}$ was detected in all isolates of B. recurrentis (data not shown). The size of the band was determined by comparison to molecular weight standards and to strain B31 of Borrelia burgdorferi sensu stricto.

PFGE. All of the strains had a linear chromosome which migrated in the gel and had a size of approximately $1 \mathrm{Mb}$. The patterns observed for $B$. recurrentis were distinct from those of tick-borne relapsing-fever and Lyme-associated borreliae (Fig. 2). All of the isolates of $B$. recurrentis carried a large plasmid of approximately $192 \mathrm{~kb}$ (range, 183 to $194 \mathrm{~kb}$ ); however, the patterns observed for 25 - to 62 -kb plasmids showed more heterogeneity. An 11-kb plasmid was present in all strains. This band was not always detectable on ethidium bromide-stained gels; however, it could be visualized following hybridization. All of the strains produced one of five plasmid patterns (Table 2 ). The presence of a major protein band belonging to group 4 (24 kDa or less) and plasmid types 3 to 5 was significantly different from the results obtained for strains that exhibited higher molecular weights for major proteins and had plasmid types 1 or 2 (chi-square test with Yates correction, 0.0009). The sizes of the plasmids found in each $B$. recurrentis group are given in Table 3. The chromosomal and plasmid bands between 25 and $194 \mathrm{~kb}$ resolved as sharp bands and thus probably represent linear DNA $(10,13)$.

DNA sequencing. The DNA sequence analysis revealed distinct signature nucleotides which distinguished Lyme-associ- 


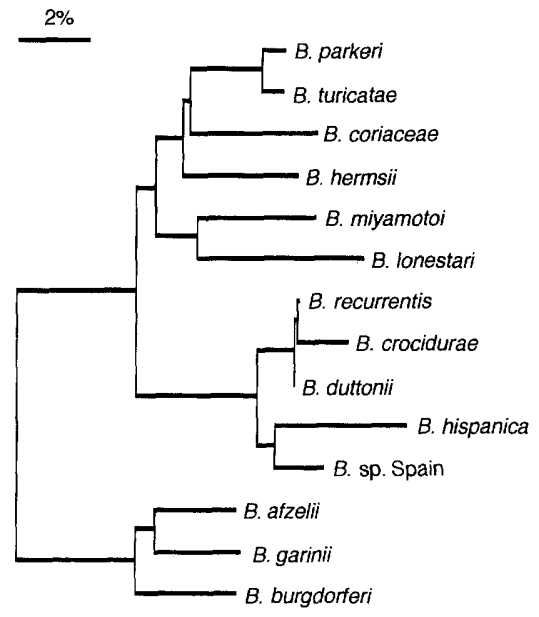

FIG. 4. Phylogenetic tree for the genus Borrelia constructed by using the neighbor-joining method and flagellin sequences.

ated borreliae from relapsing-fever borreliae; for example, there were signature nucleotides at positions 130, 193, 195, 196,287 , and 288. The relapsing-fever strains from Africa could be distinguished from those found in America by base changes at positions $65,70,190$, and 200 . B. recurrentis could be differentiated from Borrelia duttonii and indeed all of the other spirochetal strains analyzed by differences at positions 242 , 525,959 , and 1481. Interestingly, all of these differences were substitutions of thymidine for cytosine. These data are based on those of Marti Ras et al. (26). Details of the complete 16S rRNA gene sequences for relapsing-fever and Lyme-associated borreliae have been reported elsewhere (26), and the sequence of $B$. recurrentis $\mathrm{A} 1$ has been deposited in the GenBank database under accession no. U42300.

A 984-base portion of the flagellin gene was sequenced, and the results were compared with $B$. duttonii $406 \mathrm{~K}$ data. Only one base substitution was found, at position 497; adenosine was present in $B$. recurrentis, while $B$. duttonii contained a guanidine. The flagellin nucleotide sequence data have been deposited in the DDBJ, EMBL, and GenBank databases under accession no. D86618. A multiple alignment of these data with data for relapsing-fever, Lyme-associated, and other borreliae is shown in Fig. 3. A phylogenetic tree was constructed by using the neighbor-joining method, which revealed a cluster comprised of $B$. recurrentis, B. duttonii, and Borrelia crocidurae (Fig. 4 ). This cluster was more distantly related to Borrelia hispanica and the recently described Spanish relapsing-fever species (1). However, the American relapsing-fever strains clustered together and with Borrelia miyamotoi and Borrelia lonestari. The Lyme-associated species formed a third distinct cluster (Fig. 4).

$\mathbf{G}+\mathbf{C}$ content of $\boldsymbol{B}$. recurrentis DNA. The $\mathrm{G}+\mathrm{C}$ content of strain A1 was $28.4 \mathrm{~mol} \%$. This is within the range of 27 to 32 mol\% reported for members of the genus Borrelia (14).

Comparison of $B$. recurrentis DNA with DNAs from other borreliae. Both probe labelling and detection methods gave similar results for whole DNA-DNA hybridizations. B. recurrentis failed to react with the tick-borne relapsing-fever strains of Borrelia hermsii, Borrelia parkeri, and Borrelia turicatae. None of the Lyme-associated borreliae (B. burgdorferi sensu stricto, Borrelia garinii, Borrelia afzelii, Borrelia japonica, group VS116) hybridized with $B$. recurrentis, even when $200 \mathrm{ng}$ of target DNA was used. All strains of $B$. recurrentis hybridized with the probe, detecting levels of 1 to $10 \mathrm{ng}$ of DNA.
Electron microscopy. Electron microscopy revealed characteristic borrelial cells (Fig. 5a). The cells had tapering ends (Fig. 5b) and an average wavelength of $1.8 \mu \mathrm{m}$ with an amplitude of $0.8 \mu \mathrm{m}$. The length was variable, but was approximately 12 to $22 \mu \mathrm{m}$. The cells had 8 to 10 periplasmic flagella and diameters of 0.27 to $0.34 \mu \mathrm{m}$ (Fig. $5 \mathrm{c}$ through e). Flagellar counts of transverse sections revealed 10 flagella (Fig. 5c), while flagellar counts of the ends of cells revealed 8 flagella; however, insertion points could not be distinguished (Fig. 5d). Negatively stained preparations of the central portion of spirochetal cells revealed 16 flagella, and presumably the flagella were released at the point of crossover.

\section{DISCUSSION}

In general, there was similarity between the previously described morphology (20) and the electron microscopic results of this study. The cells had tapering ends (Fig. 5b) typical of those described for the genus Borrelia; however, the ends were slightly less pointed than those described for B. burgdorferi (21, 22 ). The lengths of the cells and the diameters were consistent with earlier findings (20). The wavelength of strain A1 was identical to the wavelength reported earlier (20); however, the amplitude was $0.8 \mu \mathrm{m}$, in contrast to the $0.3-\mu \mathrm{m}$ amplitude previously reported (20). Another major difference was the number of flagella. Strain A1 of B. recurrentis had between 8 and 10 periplasmic flagella. The previously studied strains were reported to possess 15 to 20 and 25 to 30 unsheathed flagella $(20,22)$. The strains described in previous studies were multiply passaged and mouse adapted, and the original country of origin was not stated. Higher numbers of flagella (15 to 26 flagella) have been observed in a newly described tick-borne relapsing-fever Borrelia species isolated from Spain (1). Strain differences may account for the difference in numbers of flagella.

It is likely that infestation by a multiplicity of lice may be a more efficient vector for relapsing fever than a single tick bite. Also, once borreliae have been introduced into a human host, there is no need for flagellum-associated attachment determinants. Hence, a possible explanation for the lower number of flagella in $B$. recurrentis than in the tick-borne strains resides in the presumption that the louse is a more efficient disease vector than the tick. Interestingly, flagellum-less mutants of $B$. burgdorferi were as capable as an isogenic flagellated strain in inducing protective antibodies in mice (29).

The dissimilarity between $B$. recurrentis and American tickborne relapsing-fever strains, Lyme-associated strains, and other borreliae was highlighted by the whole DNA-DNA hybridization studies. These studies demonstrated a high degree of similarity among the 18 culturable strains of $B$. recurrentis.

Comparison of the DNA sequence of $B$. recurrentis with the sequence of a strain of an African tick-borne relapsing-fever borrelia ( $B$. duttonii) revealed that these organisms are closely related, but differ in $4 \mathrm{bp}$ in the $16 \mathrm{~S}$ rRNA gene (26) and in 1 bp in the flagellin gene. The differences in the 16S RNA gene and the flagellin gene sequences of $B$. recurrentis and $B$. duttonii suggest that there is selective pressure to maintain the flagellin gene without mutation. Phylogenetic trees based on either the 16S RNA gene sequence or the flagellin gene sequence gave similar cluster patterns, confirming the relationship of $B$. recurrentis, $B$. duttonii, and $B$. crocidurae, which are more distantly related to relapsing-fever strains of Spanish origin. American relapsing-fever strains fell into a separate cluster together with $B$. miyamoto $i$ and $B$. lonestari, while the Lyme disease-associated borreliae clustered separately. Whether the similarity between $B$. recurrentis and $B$. duttonii reflects 


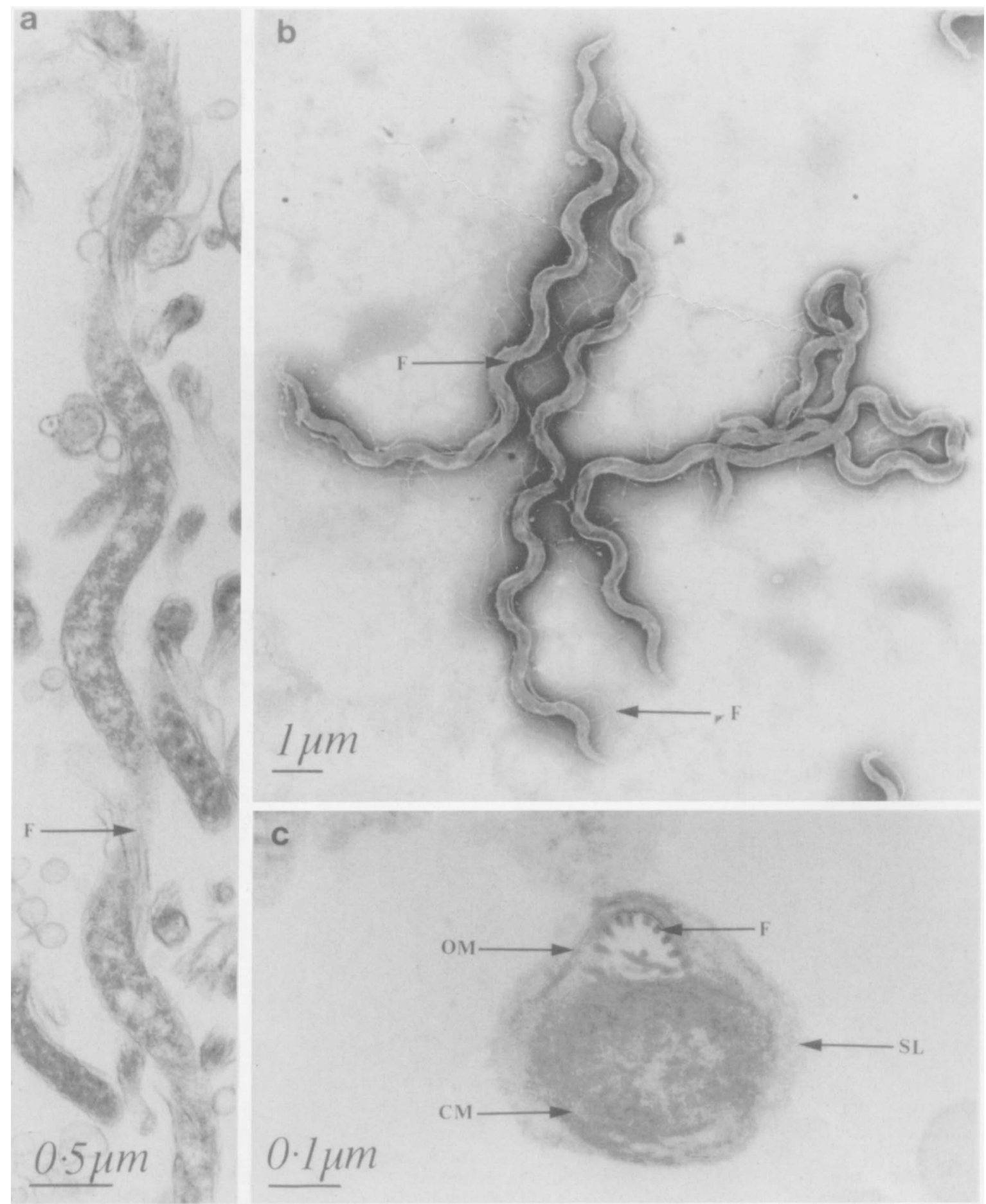

FIG. 5. Electron micrographs of B. recurrentis A1. F, flagella; SL, surface layer; OM, outer membrane; CM, cytoplasmic membrane. (a) Spirochetal morphology of cells. (b) Negative staining (phosphotungstic acid) of spirochetal cells, illustrating the tapering ends. (c) Transverse section of cell, showing the periplasmic flagella. (d) Negatively stained preparation (ammonium molybdate), showing terminally associated flagella. (e) Negatively stained preparation (ammonium molybdate), showing flagella associated with the central portion of a cell.

adaptation of the organisms to a louse vector or results from geographical or circumstantial differences remains to be resolved. There are some data suggesting that $B$. duttonii could be transmitted to a louse vector (2), but this remains to be substantiated. What is true is that the sporadic tick-borne re- lapsing fever does not seem to have the severity or epidemic potential of the louse-borne disease.

PFGE demonstrated the presence of a linear chromosome of approximately $1 \mathrm{Mb}$ in all of the strains tested. This chromosome is similar to the chromosome described for other 

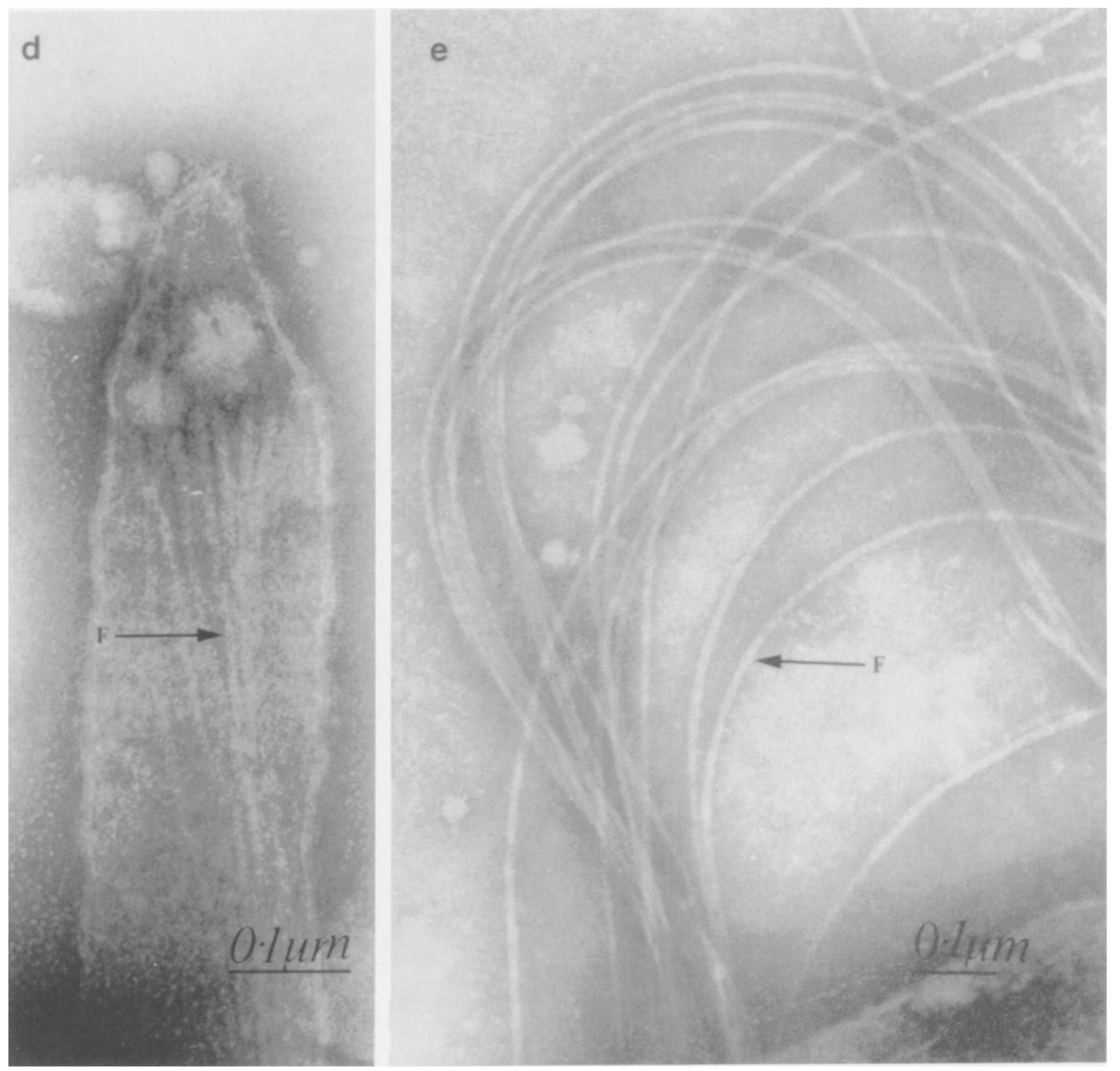

FIG. 5-Continued.

borrelial strains $(6,7,13,23)$. High-molecular-weight minichromosomes that are 180 and $170 \mathrm{~kb}$ long have been described previously in $B$. hermsii and $B$. turicatae, respectively (13), and were also demonstrated in B. recurrentis in our analysis. However, the high-molecular-weight minichromosomes in B. recurrentis were slightly larger (183 to $194 \mathrm{bp}$ ).

Both linear plasmids and linear chromosomes have been detected in relapsing-fever borreliae. Replicons with similar structures are more frequently associated with eukaryotic cells than with prokaryotes. Molecular characterization of these structures may provide clues to the evolutionary relationship between prokaryotes and eukaryotes and may also provide evidence for genetic exchange between these kingdoms (19). Workers have noted similarity between the AT-rich sequences at the ends of the specific linear plasmids of B. burgdorferi and tick-borne relapsing-fever strains and iridovirus $(8,18)$, suggesting that the linear replicons may have a common origin and may have been subject to transkingdom exchange. This is more likely as the tick is a common vector of both African swine fever (iridovirus) and relapsing fever. Since B. recurrentis is louse borne, the possible presence of these common sequences would have taxonomic and evolutionary implications, particularly in illuminating the relationship to tick-borne disease.

The serospecificity of borreliae in each clinical relapse is determined by the outer membrane proteins. The genetic mechanism of this antigenic variation has been studied in previously isolated borreliae other than $B$. recurrentis (5). It has been suggested that there is a minichromosome shuttle mechanism similar to that found with sleeping sickness trypanosomes. In B. hermsii the variable membrane lipoprotein (VMP) 
is the responsible antigen (28). Studies of $B$. hermsii have suggested three possible mechanisms of VMP variation: (i) a complete silent $v m p$ gene may replace a complete expressed $v m p$ by intermolecular recombination; (ii) a silent complete VMP gene located on another plasmid may partially replace expressed vmp (the donor VMP sequence may be an incomplete pseudogene); or (iii) the donor gene may be on the same replicon as the active $v m p$, and the serotype switch may be achieved by intramolecular recombination in the expressed plasmid (28).

Areas of homology exist at the telomeres of the different $v m p$ genes of $B$. hermsii and, indeed, have been shown to have homology with the telomeres of other relapsing-fever species, including $B$. turicatae and $B$. crocidurae (5a). Similarity has also been demonstrated between these telomeric sequences and those of other borreliae, including the OspC gene of $B$. burgdorferi sensu lato $(24,25)$ and a VMP-like gene in $B$. myamoto $i$ (17). Future use of primers for conserved telomeric sequences to amplify and sequence the VMP genes of our cultivable strains would allow us to compare these organisms with other relapsing-fever borreliae. Similarly, probes would allow us to locate the genes responsible for the antigenic variants of $B$. recurrentis. It would then be possible to characterize different antigenic variants and identify whether the differences in observed plasmid profiles seen in this study are associated with major genetic reorganizations. This has not been the case with $B$. hermsii. Both protein profiles and plasmid patterns of $B$. recurrentis allowed isolates to be grouped. The association seen in Table 2 suggests that these two events may be related. It is plausible that as strains undergo antigenic variation, the major protein responsible for serotype specificity is altered, and this event may be accompanied by a concomitant genetic reorganization, resulting in a changed plasmid profile. Up to five relapses have been reported with louse-borne relapsing fever (9); whether this correlates with the five plasmid profiles seen in Fig. 2 remains speculative.

Further study of $B$. recurrentis present in clinical material and lice and a comparison of the results with results obtained with our cultivable strains would establish whether certain culture-adapted variants are selected by our in vitro methods (a culture-associated VMP of approximately $20 \mathrm{kDa}$ has been described for $B$. hermsii [27]) and would also establish the range of VMPs expressed in primary disease (relapse variants would require the use of animal models) and the range of VMPs expressed in the louse vector.

\section{ACKNOWLEDGMENTS}

We thank the Wellcome Trust, The Royal Society, and the special trustees of Charing Cross Hospital for their assistance in making the visits to Ethiopia possible.

\section{REFERENCES}

1. Anda, P., W. Sánchez-Yebra, M. del Mar Vitutia, E. P. Pastrana, I. Rodríguez, N. S. Miller, P. B. Backenson, and J. L. Benach. 1996. A new Borrelia species isolated from patients with relapsing fever in Spain. Lancet 348:162-165.

2. Baltazard, M., M. Bahamanyar, and C. Mofidi. 1947. Fiévres Récurrentes transmises a la fois par ornithodores et par poux. Ann. Inst. Pasteur 73:10661071.

3. Barbour, A. G. 1984. Isolation and cultivation of Lyme disease spirochetes. Yale J. Biol. Med. 57:521-523.
4. Barbour, A. G., S. F. Hayes, R. A. Heiland, M. E. Schrumpf, and S. L. Tessier. 1986. A borrelia-specific monoclonal antibody binds to a flagellar epitope. Infect. Immun. 52:549-554.

5. Barbour, A. G. 1990 . Antigenic variation of a relapsing fever Borrelia species. Annu. Rev. Microbiol. 44:155-171.

5a.Barbour, A. G. 1997. Personal communication.

6. Baril, C., C. Richaud, G. Baranton, and I. S. Saint-Girons. 1989. Linear chromosome of Borrelia burgdorferi. Res. Microbiol. 140:507-516.

7. Bergström, S., A. G. Barbour, C. F. Garon, P. Hindersson, I. Saint-Girons, and T. G. Schwan. 1991. Genetics of Borrelia burgdorferi. Scand. J. Infect. Dis. Suppl. 77:102-107.

8. Bergström, S., C. F. Garon, A. G. Barbour, and J. MacDougall. 1992. Extrachromosomal elements of spirochetes. Res. Microbiol. 143:623-628.

9. Calero, C. 1946. Relapsing fever on the Isthmus of Panama. Am. J. Trop. Med. 26:761-769.

10. Casjens, S., M. Delange, H. L. Ley III, P. Rosa, and W. M. Huang. 1995. Linear chromosomes of Lyme disease agent spirochetes: genetic diversity and conservation of gene order. J. Bacteriol. 177:2769-2780.

11. Cutler, S. J., D. J. M. Wright, and V. H. Luckhurst. 1993. Simplified method for the interpretation of immunoblots for Lyme borreliosis. FEMS Immunol. Med. Microbiol. 6:281-286.

12. Cutler, S. J., D. Fekade, K. Hussein, K. A. Knox, A. Melka, K. Cann, A. R. Emilianus, D. A. Warrell, and D. J. M. Wright. 1994. Successful in-vitro cultivation of Borrelia recurrentis. Lancet 343:242. (Letter.)

13. Ferdows, M. S., P. Serwer, G. A. Griess, S. J. Norris, and A. G. Barbour. 1996. Conversion of a linear to a circular plasmid in the relapsing fever agent Borrelia hermsii. J. Bacteriol. 178:793-800.

14. Fukunaga, M., Y. Takahashi, Y. Tsuruta, O. Matsushita, D. Ralph, M. McClelland, and M. Nakao. 1995. Genetic and phenotypic analysis of Borrelia miyamotoi sp. nov., isolated from the ixodid tick Ixodes persulcatus, the vector for Lyme disease in Japan. Int. J. Syst. Bacteriol. 45:804-810.

15. Fukunaga, M., and Y. Koreki. 1996. A phylogenetic analysis of Borrelia burgdorferi sensu lato isolates associated with Lyme disease in Japan by flagellin gene sequence determination. Int. J. Syst. Bacteriol. 46:416-421.

16. Fukunaga, M., K. Okada, M. Nakao, T. Konishi, and Y. Sato. 1996. Phylogeny of Borrelia species based on flagellin gene sequences and its application for molecular typing of Lyme disease borreliae. Int. J. Syst. Bacteriol. 46: 898-905.

17. Hamase, A., Y. Takahashi, K. Nohgi, and M. Fukunaga. 1996. Homolog of variable major protein genes between Borrelia hermsii and Borrelia miyamotoi. FEMS Microbiol. Lett. 140:131-137.

18. Hinnebusch, J., and A. G. Barbour. 1991. Linear plasmids of Borrelia burgdorferi have a telomeric structure and sequence similar to those of a eukaryotic virus. J. Bacteriol. 173:7233-7239.

19. Hinnebusch, J., and K. Tilly. 1993. Linear plasmids and chromosomes in bacteria. Mol. Microbiol. 10:917-922.

20. Hovind Hougen, K. 1974. Electron microscopy of Borrelia merionesi and Borrelia recurrentis. Acta Pathol. Microbiol. Scand. Sect. B 82:799-809.

21. Hovind Hougen, K. 1984. Ultrastructure of spirochetes isolated from Ixodes ricinus and Ixodes dammini. Yale J. Biol. Med. 57:543-548.

22. Hulínská, D., J. Jirous, M. Valesová, and J. Herzogová. 1989. Ultrastructure of Borrelia burgdorferi in tissues of patients with Lyme disease. J. Basic Microbiol. 29:73-83.

23. Kitten, T., and A. G. Barbour. 1992. The relapsing fever agent Borrelia hermsii has multiple copies of its chromosome and linear plasmids. Genetics 132:311-324.

24. Marconi, R. T., D. S. Samuels, T. G. Schwan, and C. F. Garon. 1993. Identification of a protein in several Borrelia species which is related to OspC of the Lyme disease spirochetes. J. Clin. Microbiol. 31:2577-2583.

25. Margolis, N., D. Hogan, W. Cieplak, T. G. Schwan, and P. A. Rosa. 1994. Homology between Borrelia burgdorferi OspC and members of the family of Borrelia hermsii variable major proteins. Gene 143:105-110.

26. Marti Ras, N., B. Lascola, D. Postic, S. J. Cutler, F. Rodhain, G. Baranton, and D. Raoult. 1996. Phylogenesis of relapsing fever Borrelia. Int. J. Syst. Bacteriol. 46:859-865.

27. Meier, J. T., M. I. Simon, and A. G. Barbour. 1985. Antigenic variation is associated with DNA rearrangements in a relapsing fever borrelia. Cell 41:403-409.

28. Restrepo, B. I., C. J. Carter, and A. G. Barbour. 1994. Activation of a vmp pseudo gene in Borrelia hermsii: an alternative mechanism of antigenic variation during relapsing fever. Mol. Microbiol. 13:287-299.

29. Sadziene, A., P. A. Thompson, and A. G. Barbour. 1996. A flagella-less mutant of Borrelia burgdorferi as a live attenuated vaccine in the murine model of Lyme disease. J. Infect. Dis. 173:1184-1193. 